\title{
Exploring the Rotating Reference Frame in Off-Resonance Pulsed NMR
}

\author{
David B. Pengra* \\ Department of Physics, Box 351560, University of Washington, Seattle, WA 98195
}

\begin{abstract}
Understanding the physics of pulsed nuclear magnetic resonance (NMR) is eased by a transformation to a rotating reference frame. Within such a frame, coincident with the applied $\mathrm{RF} H_{1}$ field that rotates about the static $H_{0}$ field, the net effective magnetic field is static, and the dynamics of spin precession yield to simple geometric analysis. In most treatments of NMR the resonant frame, defined by the Larmor frequency $\omega_{0}=\gamma H_{0}$, is used. In this frame the effective field reduces to $H_{1}$ only, and one can immediately derive the pulse widths needed to optimize the free induction decay (FID) signals - the so-called $\pi / 2$ and $3 \pi / 2$ pulses. But what happens in a frame that is off resonance? The same geometrical analysis shows that as the frequency of $H_{1}$ is detuned from resonance, (1) the pulse width needed to maximize the FID signal increases for " $\pi / 2$ " pulses and decreases for " $3 \pi / 2$ " pulses, (2) at a critical value of detuning, the widths of both pulses converge to $\sqrt{2}$ times the width of the on-resonance $\pi / 2$ pulse, (3) this critical detuning is equal to the Larmor frequency of $H_{1}$, and (4) the maximum amplitude the FID signal is unchanged as long as the detuning is less than the critical value. Detuning further causes the optimum pulse width to decrease and the magnitude of the FID signal to drop. This experiment helps students explore a wider range of predictions inherent in the classical model of NMR, and it can be performed on the popular TeachSpin PS1 apparatus with no extra equipment.
\end{abstract}

PACS numbers: $76.60 .-\mathrm{k}$

Keywords: Off-resonance NMR, Pulsed NMR, Free induction decay

\section{INTRODUCTION}

Since the introduction in 1994 of the popular and versatile TeachSpin pulsed nuclear magnetic resonance (NMR) apparatus [1], students at many institutions can explore the physics of pulsed NMR in depth. Although NMR is a quantum phenomenon, much of the behavior can be modeled by a purely classical theory of the dynamics of particles possessing both angular momentum and a magnetic moment [2].

To review, particles with magnetic moment $\overrightarrow{\boldsymbol{\mu}}$ and angular momentum $\overrightarrow{\boldsymbol{J}}$ subject to a magnetic field $\overrightarrow{\boldsymbol{H}}_{0}$ obey an equation of motion $d \overrightarrow{\boldsymbol{\mu}} / d t=\overrightarrow{\boldsymbol{\mu}} \times \gamma \overrightarrow{\boldsymbol{H}}_{0}$, where $\overrightarrow{\boldsymbol{\mu}}=\gamma \overrightarrow{\boldsymbol{J}}$ and thus precess about the direction of $\overrightarrow{\boldsymbol{H}}_{0}$ with an angular frequency $\omega$ equal to the Larmor frequency, $\gamma H_{0}$. The quantity $\gamma$ is the gyromagnetic ratio of the particle $\left(2.675 \times 10^{8} \mathrm{rad} /(\mathrm{s}-\mathrm{T})\right.$ for a proton $)$. Call the moment plus angular momentum of an individual particle a "spin". An ensemble of spins will at equilibrium have a net magnetization $\overrightarrow{\boldsymbol{M}}$ parallel to $\overrightarrow{\boldsymbol{H}}_{0}$. This magnetization can be reoriented by the application of a pulse of a rotating magnetic field $\overrightarrow{\boldsymbol{H}}_{1}(t)$ whose direction is always perpendicular to $\overrightarrow{\boldsymbol{H}}_{0}$. During the pulse, the spins and thus $\overrightarrow{\boldsymbol{M}}$ precess about the combined field $\overrightarrow{\boldsymbol{H}}_{0}+\overrightarrow{\boldsymbol{H}}_{1}(t)$.

If $\overrightarrow{\boldsymbol{H}}_{1}(t)$ is on for a brief interval, much less than the system's characteristic relaxation time $T_{2}^{*}$ (given by $1 /(\gamma \Delta H)$ where $\Delta H$ is the spread in the static field over the sample [2, p. 40]), $\overrightarrow{\boldsymbol{M}}$ can be left in a non-equilibrium state with a substantial projection onto the $x-y$ plane. Further, if this reorientation of $\overrightarrow{\boldsymbol{M}}$ happens in the vicinity of a coil whose axis lies in the same plane, the pre- cession of $\overrightarrow{\boldsymbol{M}}$ will induce an emf in the coil that can be detected and amplified with suitable electronics before it decays. This signal is the free induction decay or FID signal [2, p. 23].

\section{The Rotating Reference Frame}

How the pulsed method creates the FID signal is shown by transforming the lab coordinate system into one that rotates about the $z$ axis with angular frequency $\omega$ equal to the angular frequency of $\overrightarrow{\boldsymbol{H}}_{1}(t)$. Within this frame, $\overrightarrow{\boldsymbol{H}}_{1}(t)$ becomes a constant, and, since the frame is rotating about the same axis as defined by $\overrightarrow{\boldsymbol{H}}_{0}$, the $z$-axis field also appears constant; however, the the magnitude of the $z$-axis field is not the same as in the lab frame.

This can be understood as follows. First let $\overrightarrow{\boldsymbol{H}}_{1}(t)$ be represented by

$$
\overrightarrow{\boldsymbol{H}}_{1}(t)=H_{1} \cos (\omega t) \hat{\mathbf{i}}-H_{1} \sin (\omega t) \hat{\mathbf{j}}
$$

This field lies in the $x-y$ plane and rotates clockwise about the $z(\hat{\mathbf{k}})$ direction. Let new unit vectors $\hat{\mathbf{i}}^{\prime}$ and $\hat{\mathbf{j}}^{\prime}$ be given by

$$
\begin{aligned}
& \hat{\mathbf{i}}^{\prime}=\hat{\mathbf{i}} \cos (\omega t)-\hat{\mathbf{j}} \sin (\omega t), \\
& \hat{\mathbf{j}}^{\prime}=\hat{\mathbf{i}} \sin (\omega t)+\hat{\mathbf{j}} \cos (\omega t) .
\end{aligned}
$$

By comparing Eq. (2) to Eq. (1) we see that $\overrightarrow{\boldsymbol{H}}_{1}(t)$ may be written $H_{1} \hat{\mathbf{1}}^{\prime}$.

What happens to $\overrightarrow{\boldsymbol{H}}_{0}$ ? First, consider the resonant case where the rotation frequency $\omega=\omega_{0}=\gamma H_{0}$ (the 
Larmor frequency associated with the static field) and for the moment assume $H_{1}=0$. Within this frame one would see no precession: by construction one is "riding along" with the spins; hence, it appears than $\overrightarrow{\boldsymbol{H}}_{0}=0$ in this frame. In a frame where the rotation frequency is not equal to $\omega_{0}$, spins would appear to precess at an apparent frequency of $\omega_{0}-\omega$. The sign of this difference is significant: in a frame where $\omega>\omega_{0}$, the reference frame rotates faster than the spin precession, and the apparent precession would rotate oppositely to the direction seen in the lab frame. These observations suggest that within the rotating frame of any $\omega$, the effective static field $\overrightarrow{\boldsymbol{H}}_{0}$ becomes $\left(H_{0}-\omega / \gamma\right) \hat{\mathbf{k}}$.

Thus, the net field in the rotating frame is given by

$$
\overrightarrow{\boldsymbol{H}}_{\mathrm{eff}}=\left(H_{0}-\frac{\omega}{\gamma}\right) \hat{\mathbf{k}}+H_{1} \hat{\mathbf{i}}^{\prime},
$$

a result proved rigorously in texts on NMR [2, Ch. 2].

On resonance, $H_{0}-\omega / \gamma=0$ and we are left with only $H_{1} \hat{\mathbf{\imath}}^{\prime}$ in the rotating frame. One can easily see what a pulse of $H_{1} \hat{\mathbf{i}}^{\prime}$ would accomplish: a rotation about $\hat{\mathbf{i}}^{\prime}$ with an angular frequency of $\gamma H_{1}$. By applying a pulse of duration

$$
t_{\pi / 2}=\frac{\pi}{2}\left(\frac{1}{\gamma H_{1}}\right)
$$

$\overrightarrow{\boldsymbol{M}}$ tips by $90^{\circ}$ or $\pi / 2$ giving maximum projection of $\overrightarrow{\boldsymbol{M}}$ onto the $x-y$ plane. Another maximum in the FID signal occurs with a pulse of 3 times $t_{\pi / 2}$ that rotates $\overrightarrow{\boldsymbol{M}}$ by $270^{\circ}$ : a " $3 \pi / 2$ pulse."

Once the above results are derived, students may not realize that the coordinate transformation is not a mere mathematical trick to obtain Eq. (5), but that the general result of Eq. (4) leads to a number of other experimentally testable predictions regarding the FID signal, which we now consider.

\section{PRECESSION DYNAMICS OFF-RESONANCE}

Off-resonance, $\overrightarrow{\boldsymbol{H}}_{\text {eff }}$ no longer lies in the $x^{\prime}-y^{\prime}$ plane, but tilts away from it. Consequently, when a pulse is applied $\overrightarrow{\boldsymbol{M}}$ precesses about a cone with apex defined by $\overrightarrow{\boldsymbol{H}}_{\text {eff }}$ and $\hat{\mathbf{k}}$. The geometry of this situation is shown in Figure 1.

It is convenient to express Eq. (4) in terms of the amount of detuning $\Delta \omega=\omega_{0}-\omega$. By definition, $H_{0}-\omega_{0} / \gamma=0$, thus we may write $\overrightarrow{\boldsymbol{H}}_{\text {eff }}=\Delta \omega / \gamma \hat{\mathbf{k}}+H_{1} \hat{\mathbf{\imath}}^{\prime}$. The angle $\alpha$ and field magnitude $H_{\text {eff }}$ are given by

$$
\begin{aligned}
\tan \alpha & =\frac{\Delta \omega}{\gamma H_{1}} \\
H_{\mathrm{eff}} & =H_{1} \sqrt{1+\left(\frac{\Delta \omega}{\gamma H_{1}}\right)^{2}} .
\end{aligned}
$$

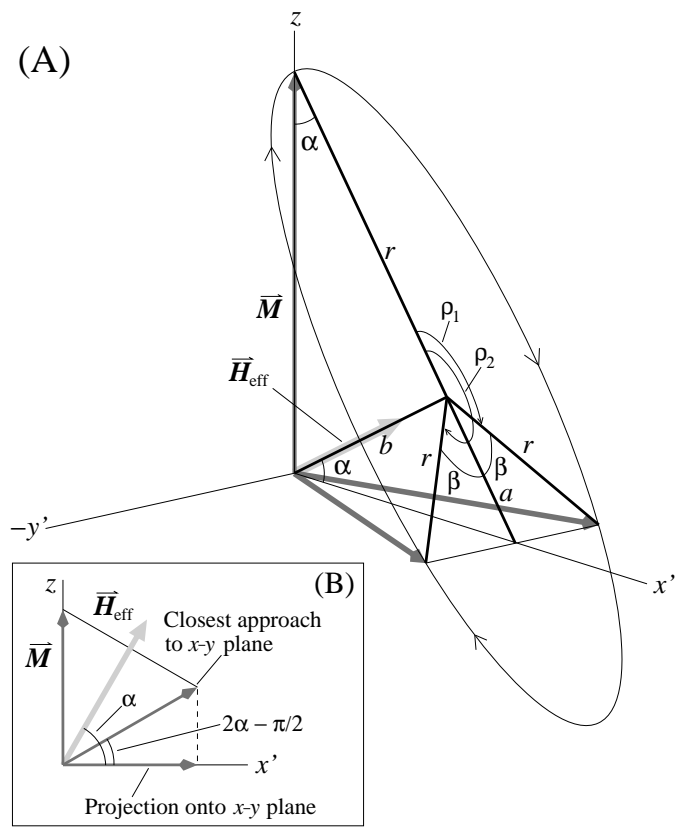

FIG. 1: The geometry of precession for an $H_{1}$ field detuned from resonance. (A) With detuning $|\Delta \omega|<\gamma H_{1}$ intersection of the $x^{\prime}-y^{\prime}$ plane is possible through rotations $\rho_{1}$ or $\rho_{2}$. (B) Detuning above $\gamma H_{1}$ a $\pi$ pulse gives the angle of closest approach of $\overrightarrow{\boldsymbol{M}}$ to the $x^{\prime}$ axis. Symbols are defined in the text.

As shown in Fig. 1-A, if $|\Delta \omega|<\gamma H_{1}$, the base of the cone traced out by the tip of $\overrightarrow{\boldsymbol{M}}$ still intersects the $x^{\prime}-y^{\prime}$ plane. Thus, a suitable pulse width exists that should produce the same amplitude FID signal as would be found on-resonance. Note further that the rotation angle $\rho_{1}$ needed to make the first intersection of $\overrightarrow{\boldsymbol{M}}$ with this plane is greater than $\pi / 2$, but the angle $\rho_{2}$ needed to make the second intersection is less than $3 \pi / 2$.

At the critical value of detuning, where $|\Delta \omega|=\gamma H_{1}$, the angle $\alpha=\pi / 4$ and the base of the precession cone just touches the $x^{\prime}$ and $z$ axes. Now both $\rho_{1}$ and $\rho_{2}$ have converged to become exactly $\pi$.

With further detuning, $\overrightarrow{\boldsymbol{M}}$ no longer passes through the $x^{\prime}-y^{\prime}$ plane. The closest approach to the plane will occur with a $\pi$ rotation, and the magnitude of the FID signal will decrease, being proportional to the projection of $\overrightarrow{\boldsymbol{M}}$ onto the plane, as indicated by Fig. 1-B.

In addition, as $|\Delta \omega|$ increases, $\gamma H_{\text {eff }}$ increases, so the precession speed increases. The pulse width needed to achieve rotation through a particular angle will decrease with detuning proportional to $\left[1+\left(\Delta \omega / \gamma H_{1}\right)^{2}\right]^{-1 / 2}$.

Taken together, the change in $\rho_{1}$ or $\rho_{2}$ plus the increase in the precession speed determines the optimum pulse width needed to maximize the FID signal. The mathematical form of pulse width versus detuning is treated below, but it is already apparent that at the critical value 
where $|\Delta \omega| /\left(\gamma H_{1}\right)=1, H_{\text {eff }}=\sqrt{2} H_{1}$ and $\rho_{1}=\rho_{2}=\pi$, the width of the pulse at critical detuning $t_{c}=\sqrt{2} t_{\pi / 2}$.

\section{Qualitative Predictions Off-Resonance}

Without any further analysis, the following predictions can be immediately tested by students using the TeachSpin PS1 apparatus or its equivalent. Let $t_{1}$ and $t_{2}$ be the pulse widths needed to achieve rotations through $\rho_{1}$ and $\rho_{2}$, both of which maximize the FID signal. As the oscillator that creates the $\overrightarrow{\boldsymbol{H}}_{1}(t)$ field is tuned away from resonance

- $t_{1}$ will increase and $t_{2}$ will decrease;

- the rate of decrease in $t_{2}$ will be more rapid than the rate of increase in $t_{1}$, because detuning decreases $\rho_{2}$ but increases $\rho_{1}$ while simultaneously decreasing the time needed to achieve either rotation;

- at a pulse width equal to $\sqrt{2} t_{\pi / 2}$ the amount of detuning $\Delta \omega$ needed to maximize the FID signal is equal to the Larmor frequency associated with $H_{1}$;

- no change in the amplitude of the maximum FID signal should be observed until $|\Delta \omega|$ is greater than $\gamma H_{1}$;

- further detuning should result in a monotonic decrease in both the optimum pulse width needed to achieve maximum FID signal and the magnitude of the FID signal itself.

\section{Quantitative Analysis}

The functional forms of the pulse widths $t_{1}$ and $t_{2}$, plus the maximum FID amplitude as a function $\Delta \omega$, may be derived from the geometry shown in Fig. 1-A. Let $r$ be the radius of the cone base, $a$ be the line segment from the cone axis to the $x^{\prime}$ axis, and $b$ be the line segment from the cone apex to the cone base. The angle $\alpha$ is as defined in Eq. (6), and the angle $\beta$ is the angle between line segment $a$ and the radial lines associated with the magnetization vector $\overrightarrow{\boldsymbol{M}}$ after it has rotated through angles $\rho_{1}$ and $\rho_{2}$. By construction $\cos \beta=a / r$ and $\tan \alpha=a / b=b / r$. Thus, $a=b^{2} / r$ and $a / r=(b / r)^{2}$, which gives the relation $\cos \beta=\tan ^{2} \alpha$. Since $\rho_{1}=\pi-\beta$ and $\rho_{2}=\pi+\beta$, we have

$$
\begin{aligned}
& \rho_{1}=\pi-\cos ^{-1}\left[\left(\frac{\Delta \omega}{\gamma H_{1}}\right)^{2}\right], \\
& \rho_{2}=\pi+\cos ^{-1}\left[\left(\frac{\Delta \omega}{\gamma H_{1}}\right)^{2}\right] .
\end{aligned}
$$

The pulse widths $t_{1}$ and $t_{2}$ must satisfy the equations $\rho_{1}=\gamma H_{\text {eff }} t_{1}$ and $\rho_{2}=\gamma H_{\text {eff }} t_{2}$ when $|\Delta \omega|<\gamma H_{1}$, and satisfy $\pi=\gamma H_{\text {eff }} t_{1,2}$ thereafter. It is useful to express the final equations in terms of the detuning frequency in hertz $\Delta f=\Delta \omega / 2 \pi$ and a characteristic time $\tau=2 \pi /\left(\gamma H_{1}\right)$ (i.e., time for a $2 \pi$ rotation on resonance), which yield after a little algebra

$$
\begin{aligned}
& t_{1}=\frac{\tau\left(1-\frac{1}{\pi} \cos ^{-1}\left[(\tau \Delta f)^{2}\right]\right)}{2 \sqrt{1+(\tau \Delta f)^{2}}}, \\
& t_{2}=\frac{\tau\left(1+\frac{1}{\pi} \cos ^{-1}\left[(\tau \Delta f)^{2}\right]\right)}{2 \sqrt{1+(\tau \Delta f)^{2}}} .
\end{aligned}
$$

It is easy to show that on resonance, when $\Delta f=0$, $t_{1}=\tau / 4$ and $t_{2}=3 \tau / 4$, the times that produce a $\pi / 2$ pulse and $3 \pi / 2$ pulse, respectively. At the critical value $\tau \Delta f=1, t_{1}=t_{2}=t_{c}=\tau /(2 \sqrt{2})$, as expected.

For $|\tau \Delta f|>1$ the optimum pulse width is given by the simpler form

$$
t_{1,2}=\frac{\tau}{2 \sqrt{1+(\tau \Delta f)^{2}}} .
$$

The functional form of the amplitude of the maximum FID signal is also easily derived. Figure 1-B shows the situation. Since one edge of the cone lies along the $z$ axis, the angle of closest approach of $\overrightarrow{\boldsymbol{M}}$ is $2 \alpha-\pi / 2$ for $\alpha>0,2 \alpha+\pi / 2$ for $\alpha<0$. (Note $\alpha<0$ when $\Delta f<0$.) When the detuning is below the critical value such that $|\tau \Delta f|<1$, then the maximum FID amplitude is a constant; call this $A_{0}$. Above critical detuning, the maximum amplitude should be proportional to the projection of $\overrightarrow{\boldsymbol{M}}$ onto the $x^{\prime}-y^{\prime}$ plane, which is $A_{0} \cos (2|\alpha|-\pi / 2)$. By some manipulation of trigonometric identities, one can show that $\cos (2|\alpha|-\pi / 2)=2|\tan \alpha| /\left(1+\tan ^{2} \alpha\right)$. Since $\tan \alpha=\tau \Delta f$, we obtain

$$
A(\Delta f)= \begin{cases}A_{0} & \text { if }|\Delta f|<1 / \tau \\ A_{0}\left(\frac{2|\tau \Delta f|}{1+(\tau \Delta f)^{2}}\right) & \text { otherwise }\end{cases}
$$

\section{EXPERIMENTAL RESULTS}

Measurements of $t_{1}, t_{2}$ and FID maximum amplitude $A$ were done using a TeachSpin PS1-A Pulsed NMR Spectrometer [1]. This unit can create an $H_{1}$ field of $\approx 10$ gauss, giving a $\pi / 2$ pulse of $\approx 5 \mu \mathrm{s}$. The sample was mineral oil with $T_{1}$ and $T_{2}$ relaxation times of about $30 \mathrm{~ms}$. The resonant frequency of protons in our apparatus $f_{0}=14.87 \pm 0.01 \mathrm{MHz}$, which drifts due to temperature sensitivity of the permanent magnet. The FID pulse following the application of the $H_{1}$ pulse has a width of 


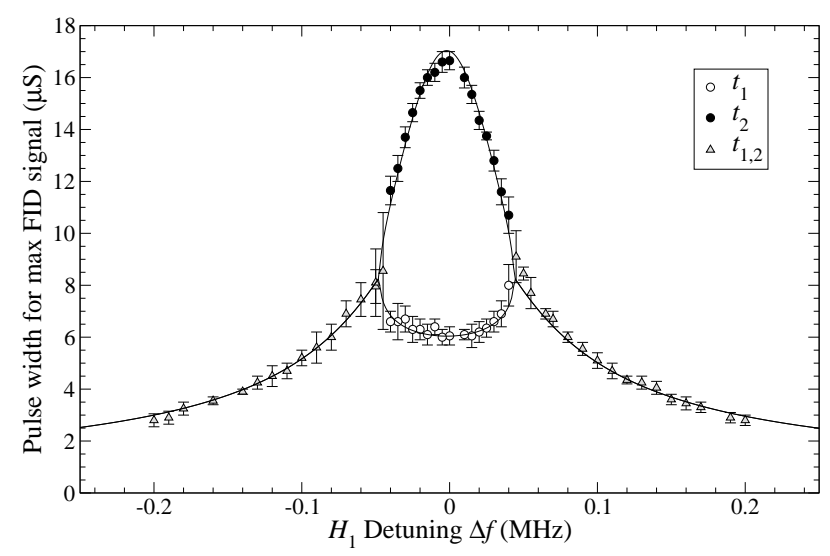

FIG. 2: Pulse widths needed to produce the maximum FID signal as a function of the detuning from resonance. On resonance, $t_{1}=t_{\pi / 2}$ and $t_{2}=t_{3 \pi / 2}$. Critical detuning occurs when $t_{1}=t_{2}$. Error bars indicate the range of no observable change in FID maximum for a given value of detuning. Lines through data are Eqs. (10)-(12).

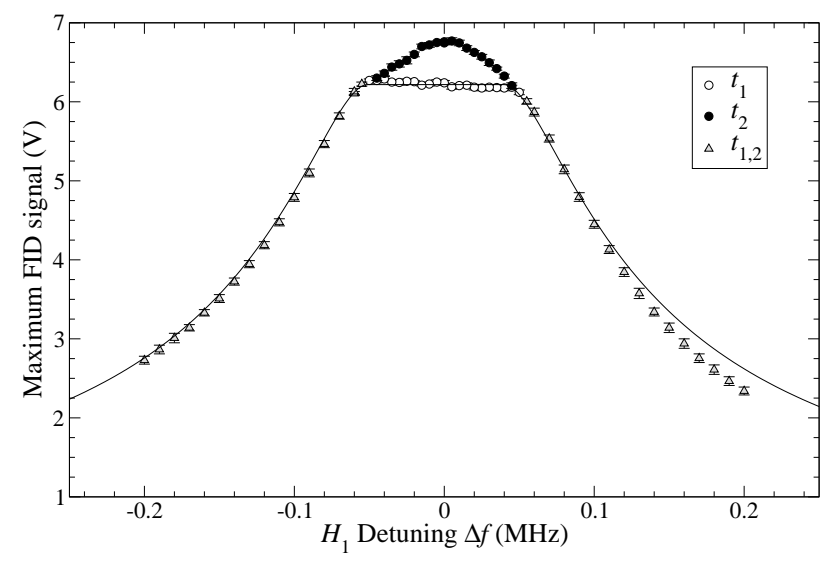

FIG. 3: Maximum FID signal as a function of the detuning from resonance. Critical detuning occurs at point where FID signal drops. Error bars indicate measurable fluctuations in maximum FID signal for a given value of detuning. Line through data is Eq. (13).

$\approx 200 \mu \mathrm{s}$. Frequency measurements were read from the RF oscillator display. Pulse widths and FID maxima were measured with a Tektronix TDS3012 digital oscilloscope.

Results are shown in Figures 2 and 3. The qualitative predictions of the model are clearly evident: the convergence of $t_{1}$ and $t_{2}$ at a critical detuning, the subsequent monotonic drop in $t_{1,2}$ beyond that, and the accompanying constant FID amplitude below critical detuning (for $t_{1}$ pulses; see below) and drop in amplitude beyond.

The most difficulty in taking measurements occurs when $\Delta f$ is close to the critical value. In this region the FID signal does not drop much between $t_{1}$ and $t_{2}$ values, but dips to a local minimum that disappears as critical detuning is approached.

The curves given by Eqs. (10)-(13) are shown in Figs. 2 and 3. $\tau=22.0 \mu \mathrm{s}$ in all curves, corresponding to $t_{\pi / 2}=5.5 \mu \mathrm{s}$ or $H_{1}=10.7$ gauss. Fits to each equation separately give a spread in $\tau$ of $\pm 1 \mu \mathrm{s}$, thus the uncertainty in $H_{1}$ is about 0.5 gauss.

The data reveal some notable systematic effects not treated by the theory. First, measured values of $t_{1,2}$ are always above the one-parameter curve given by Eq. (12) regardless of the value of $\tau$, which suggests a disparity between the pulse width supplied by the pulse generator and the RF pulse delivered to the sample. Examination of the RF signal with a coil inserted into the sample holder shows not a square pulse but one that rises and decays following two exponential curves: a rise with a time constant of $0.66 \pm 0.04 \mu$ s and decay with a time constant of $0.32 \pm 0.02 \mu \mathrm{s}$. Also, the time between the leading and falling edges of the RF pulse is slightly shorter than input TTL pulse by $0.03 \pm 0.01 \mu \mathrm{s}$. Combined, these effects shorten the applied pulse by $0.37 \pm 0.05 \mu \mathrm{s}$. The theoretical curves shown in Fig. 2 have a vertical offset of $0.54 \mu \mathrm{s}$, which is more than the directly measured $0.37 \mu \mathrm{s}$. This may indicate additional pulse-shortening effects.

The amplitude data show two systematic differences from the theory whose cause is still unknown. First, the FID amplitude following $t_{2}$ (i.e., " $3 \pi / 2$ ") pulses is greater than following $t_{1}$ pulses, as is evident in Fig. 3. Second, there is a notable asymmetry in the amplitude measurements, with the amplitudes at positive detuning being less than those at negative detuning.

TeachSpin reports that the difference in time constants between rise and fall of the RF pulse is due to a shift in the impedance of the line driving the $H_{1}$ coil when it switches from the on state to the off state, which also causes a shift in the RF frequency during the decay. These effects are being investigated to determine whether they could give rise to the systematic shifts seen in the amplitude data. A test with a newer TeachSpin PS2 apparatus, which has improved coupling in the $H_{1}$ circuit, shows no FID amplitude difference between on-resonance $\pi / 2$ and $3 \pi / 2$ pulses.

I am very grateful for conversations with Jonathan Reichert and George Herold at TeachSpin, Inc.

* dbpengra@uw.edu

[1] TeachSpin, Inc., Tri-Main Center-Suite 409, 2495 Main Street, Buffalo, NY 14214-2153. www.teachspin.com

[2] C. P. Slichter, Principles of Magnetic Resonance, third edition, Springer Series in Solid-State Sciences, Vol. 1. (Springer, New York, 1996). 